\title{
Avaliação do Plano de Gerenciamento de Resíduos dos Serviços de Saúde em uma rede de Drogarias de Vitória da Conquista-BA
}

\author{
Rafael Diego Cardoso Marques Oliveira ; Jaqueline Ferraz Rodrigues Coqueiro²; \\ Fernanda Santos Portela 3 : Monique Alves Ferraz.
}

\begin{abstract}
Resumo: Este estudo aborda o gerenciamento de resíduos de serviços de saúde em uma rede de drogarias no município de Vitória da Conquista na Bahia. Metodologia: Trata-se de uma pesquisa de caráter descritivo, com delineamento transversal mediante uma abordagem qualitativa e quantitativa. Os dados foram coletados através da aplicação de um questionário estruturado e adaptado na legislação vigente de acordo com a RDC 306/2004 da ANVISA e a RDC 358/2005 do CONAMA. Resultados: Pelo estudo realizado e de acordo com as normas vigentes, foi possível observar que as três drogarias analisadas, produz resíduos que são classificados como resíduos do grupo A biológico, grupo B químico, grupo D comuns e do grupo E perfurocortantes. Verificou-se que todas as drogarias possuem um Plano de Gerenciamento de Resíduos de Serviços de Saúde (PGRSS) e que o manejo interno dos resíduos é realizado de maneira adequada. As práticas realizadas quanto a identificação, segregação, acondicionamento, transporte, acondicionamento final e o abrigo de resíduos estão em concordância com as legislações vigentes. Conclusão: O gerenciamento de resíduos dos serviços de saúde na área do estudo apresentaram resultados satisfatórios conforme preconizados pela legislação.
\end{abstract}

Palavras-chave: Serviços de Saúde, Legislação Sanitária, Riscos Ocupacionais.

\section{Evaluation of the Health Services Waste Management Plan in a network of drugstores in Vitória da Conquista-BA}

\begin{abstract}
The present study addresses the management of health care waste in a drugstore chain in the city of Vitória da Conquista in Bahia. Methodology: This is a descriptive research, with a cross-sectional design through a qualitative and quantitative approach. The data were collected through the application of a questionnaire structured and adapted in the current legislation according to RDC 306/2004 of ANVISA and RDC 358/2005 of CONAMA. Results: It was possible to observe that the three drugstores analyzed produced residues that are classified as group A biological, group B chemical, group D common and group E piercing. It was verified that all drugstores have a Health Services Waste Management Plan (PGRSS) and that the internal waste management is carried out in an adequate manner. The practices carried out regarding identification, segregation, packaging, transport, final packaging and the shelter of waste are in accordance with the current legislation. Conclusion: Waste management of health services in the study area presented satisfactory results as recommended by the legislation.
\end{abstract}

Keywords: Health Services, health Legislation, Occupational Risks.

${ }^{1}$ Graduando do curso de Farmácia pela Faculdade Independente do Nordeste - FAINOR. E-mail: rafael_diego15@hotmail.com;

${ }^{2}$ Graduação em Farmácia pela Universidade Tiradentes, Mestre em Planejamento Ambiental pela Universidade Católica de Salvador, UCSAL. Especialista em Saúde Pública com complementação em Magistério Superior. Especialista em Saúde Mental pela Universidade Federal do Rio de Janeiro e em MBA em Auditoria em Serviços de Saúde. Atualmente é Farmacêutica da Vigilância Sanitária da Prefeitura Municipal de Vitoria da Conquista. BA. Coordenadora do Curso de Farmácia da Faculdade Independente do Nordeste - FAINOR;

${ }^{3}$ Graduação em Farmácia pela Faculdade Independente do Nordeste - FAINOR, Pós-graduada em análises clinicas e toxicológicas pela FAINOR; Pós-graduanda em farmacologia clinica com ênfase em prescrição farmacêutica pelo IBRAS;

${ }^{4}$ Graduanda do curso de Farmácia pela Faculdade Independente do Nordeste - FAINOR. E-mail: monny-alves@ @otmail.com. 


\section{Introdução}

Os estabelecimentos de saúde se configuram como locais públicos ou privados que prestam serviços de assistência à saúde, como, prevenção, acompanhamento, diagnóstico e tratamento de diversas condições clínicas. Nestes locais são executadas atividades geradoras de resíduos que, de acordo com as normas brasileiras, é necessário um manejo adequado conforme as características dos resíduos produzidos, que podem ser originados por serviços médicos, odontológicos, farmacêuticos, veterinários e laboratoriais e são chamados de resíduos de serviços de saúde (BRASIL, 2004).

De acordo com a RDC 306/2004 da Agência Nacional de Vigilância Sanitária, ANVISA e a resolução 358/2005 do Conselho Nacional de Meio Ambiente CONAMA são recomendáveis que estes locais possuam o plano de gerenciamento de resíduos de serviços de saúde (PGRSS). Este documento visa implantar e desenvolver ações sistematizadas baseados no tipo e características dos resíduos produzidos, a fim de minimizar riscos ocupacionais no ambiente de trabalho e proteger a saúde do trabalhador e da população em geral (BRASIL, 2004,2005). A principal lei regulamentadoras vigente no Brasil voltada para o manejo interno do gerenciamento de resíduos de serviços de saúde está a RDC nº 306 07/12/2004 da ANVISA que classifica em resíduos potencialmente infectantes do grupo A, resíduos com risco químico do grupo $\mathrm{B}$, rejeitos radioativos do grupo $\mathrm{C}$, resíduos comuns e recicláveis do grupo D e materiais perfurocortantes do grupo E (BRASIL, 2004).

Algumas das ações preconizadas são as identificações, acondicionamento dos resíduos, tratamento e disposição final, os quais permitem diminuir os impactos ambientais e sociais (BRASIL, 2004,2005). A RDC 306/2004 é a principal lei regulamentadora, vigente no Brasil, voltada para o manejo interno do gerenciamento de resíduos de serviços de saúde, e esta lei os classificam em: potencialmente infectantes (grupo A), resíduos com risco químico (grupo B), rejeitos radioativos (grupo $\mathrm{C}$ ), resíduos comuns e recicláveis (grupo D) e materiais perfurocortantes (grupo E) (BRASIL, 2004).

A Resolução no 358 de 29/04/2005 do CONAMA, por sua vez, estabelece o manejo externo que vai do tratamento até a disposição final dos resíduos visando preservação dos recursos naturais e do meio ambiente. (BRASIL, 2005). Enquanto que a lei $\mathrm{n}^{\mathrm{o}} 12.305$ de 02 de agosto de 2010, institui a Política Nacional de Resíduos Sólidos (PNRS) para reduzir os 
problemas ambientais decorrentes do manejo inadequado dos resíduos o Brasil (BRASIL, 2010).

Assim, diante do aspecto ambiental o destino inadequado dos resíduos afeta de forma significativa o meio ambiente através da contaminação das águas, do solo e do ar. Deve-se considerar neste contexto, também, que medicamentos não utilizados e/ou vencidos são descartados por grande parte da população em locais impróprios, geralmente em lixo comum ou jogados na pia ou vaso sanitário (Bueno, 2009; Maia; Giordano, 2012).

Diante dessa problemática devem ser estabelecidos critérios para alertar a população a respeito do uso racional de medicamentos (URM), além do seu impacto quando descartados no meio ambiente de maneira inadequada. Ademais, é imprescindível a adoção de ações bem esclarecidas acerca do destino do resíduo gerado e a redução de sua geração, visto que esses resíduos merecem uma atenção desde a sua produção até a sua disposição final, pois são resíduos perigosos e exigem cuidados técnicos, para não acarretar riscos à saúde pública (Maia; Giordano, 2012).

Essa pesquisa apresenta como objetivo geral avaliar o manejo interno dos resíduos produzidos em uma rede de drogarias no interior da Bahia de acordo com as normas brasileiras vigentes, e como objetivo especifico verificar as etapas do manejo interno desses resíduos, se estão de acordo com os critérios técnicos da legislação.

Esse trabalho é relevante para a sociedade, pois permitirá com que as pessoas tenham o conhecimento sobre o gerenciamento dos resíduos, assim como dos riscos e dos efeitos negativos causados ao meio ambiente uma vez que esses resíduos são descartados em locais inapropriados.

\section{Metodologia}

Trata-se de uma pesquisa de caráter descritivo, com delineamento transversal mediante uma abordagem qualitativa e quantitativa. O local de estudo foi em uma rede de drogarias localizada na região sudoeste no interior da Bahia na cidade de Vitória da Conquista, onde possui três estabelecimentos localizados em diferentes bairros da cidade. 
Os dados foram coletados através da aplicação de um questionário e de análise observacional, respondido e acompanhado pelo profissional farmacêutico responsável pelo estabelecimento gerador dos resíduos. O questionário continha perguntas objetivas, que foram estruturadas e adaptadas na legislação vigente de acordo com a RDC 306/2004 e a RDC 358/2005, que determinam quais os aspectos obrigatórios que cada gerador de Resíduos de Serviços de Saúde deve cumprir. Em seguida os dados obtidos foram organizados em um banco de dados eletrônico (Microsoft excel).

A análise teve como objetivo avaliar o manejo dos resíduos dos serviços de saúde do grupo A (resíduos biológicos), B (resíduos químicos), D (resíduos comuns), E (resíduos perfurocortantes), bem como as etapas de execução do processo (segregação, acondicionamento, identificação, armazenamento, transporte, tratamento e disposição final) produzidas pelos estabelecimentos em estudo.

\section{Resultados e Discussão}

Conforme os objetivos da pesquisa buscou-se analisar a presença efetiva do Plano de Gerenciamento de Resíduos de Serviços de Saúde (PGRSS) em farmácias localizadas em diferentes bairros da cidade de Vitória da Conquista - Bahia. O estudo gerou resultados que foram analisados e descritos no gráfico e quadros abaixo, além de uma proposta da estrutura de um Plano de Gerenciamento de Resíduos de Serviços de Saúde.

Um dado que pode ser notado nas três empresas avaliadas, foi que as mesmas possuem o PGRSS e as põem em prática de acordo com as normas exigidas. O PGRSS contempla itens como: a segregação dos resíduos, o acondicionamento, a identificação, o armazenamento, o transporte interno e o transporte externo, até a devida coleta.

Um estudo realizado por Moro (2017), em Fortaleza - CE mostrou que não são todas as farmácias entrevistadas que possui um plano de gerenciamento de resíduos de serviço de saúde, no entanto, as empresas que realizam esse plano apresentam falhas no manejo dos resíduos. Dessa forma, concluiu-se que há uma deficiência na capacitação e treinamento entre os funcionários dos estabelecimentos avaliados. 
Visto os dados apresentados até agora, o gráfico 1 mostra os tipos de resíduos (biológico, químico, radioativo, comum e perfurocortantes) e a quantidade em $\mathrm{kg}$ de resíduos diários gerados por cada empresa, distinguidos em empresa 1, empresa 2 e empresa 3.

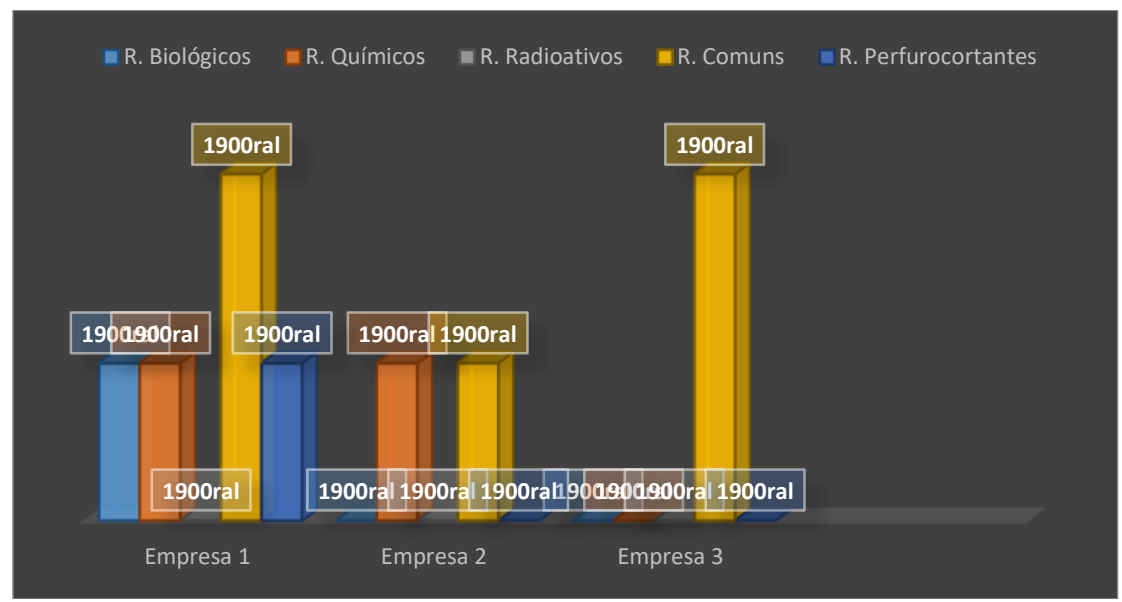

Gráfico 1: Tipos de resíduos (kg) gerados pelas empresas.

Como pode ser observado no gráfico acima, foram analisados a geração de resíduos produzidos pelas três empresas estudadas. Os resultados demonstram que a empresa 1 gera em média $13 \mathrm{~kg}$ de resíduos diários, enquanto que a empresa 3 gera $5,5 \mathrm{~kg}$ e a empresa 2 gera $5 \mathrm{~kg}$, representando um grande volume de descarte diário.

De acordo com RDC n 306 , de 7 de dezembro de 2004 da ANVISA, todas as farmácias, independente do porte, estão enquadradas como geradores de Resíduos de Serviço de Saúde (RSS).

Um estudo realizado no estado do Rio Grande do Sul verificou-se uma coleta de 5.460 toneladas de RSS, caracterizando um aumento em torno de 289 toneladas comparadas com o ano de 2013 (ABRELPE, 2014). Das empresas investigadas no presente estudo, apenas uma tem um valor mínimo de resíduos, fazendo descarte apenas de resíduos comuns.

De acordo com o estudo realizado, o manejo dos RSS pode ser evidenciado conforme o quadro 1, que demonstra a segregação e acondicionamento que é realizado por cada empresa. 
Quadro 1. Manejo dos resíduos gerados pelas empresas.

\begin{tabular}{|l||l|l||l||}
\hline \multicolumn{1}{|c||}{$\begin{array}{c}\text { Etapas do manejo dos } \\
\text { RSS }\end{array}$} & \multicolumn{1}{|c|}{ Empresa 1 } & \multicolumn{1}{|c|}{ Empresa 2 } & \multicolumn{1}{|c|}{ Empresa 3 } \\
\hline $\begin{array}{l}\text { Segregação dos resíduos } \\
\text { biológicos }\end{array}$ & $\begin{array}{l}\text { É realizada na } \\
\text { drogaria }\end{array}$ & Não é realizada & Não é realizada \\
\hline $\begin{array}{l}\text { Acondicionamento dos } \\
\text { resíduos biológicos }\end{array}$ & $\begin{array}{l}\text { São acondicionados } \\
\text { em sacos plásticos } \\
\text { de cor branca }\end{array}$ & $\begin{array}{l}\text { Não tem } \\
\text { conhecimento }\end{array}$ & Não é realizada \\
\hline $\begin{array}{l}\text { Segregação dos resíduos } \\
\text { químicos }\end{array}$ & $\begin{array}{l}\text { É segregado na } \\
\text { drogaria }\end{array}$ & $\begin{array}{l}\text { É segregado na } \\
\text { drogaria }\end{array}$ & Não é realizada \\
\hline $\begin{array}{l}\text { Acondicionamento dos } \\
\text { resíduos químicos }\end{array}$ & $\begin{array}{l}\text { Em recipientes de } \\
\text { plástico }\end{array}$ & $\begin{array}{l}\text { Não tem } \\
\text { conhecimento }\end{array}$ & Não é realizada \\
\hline $\begin{array}{l}\text { Segregação dos rejeitos } \\
\text { radioativos }\end{array}$ & Não é realizada & Não é realizada & Não é realizada \\
\hline $\begin{array}{l}\text { Acondicionamento dos } \\
\text { rejeitos radioativos }\end{array}$ & Não produz & Não produz & Não produz \\
\hline $\begin{array}{l}\text { Segregação dos resíduos } \\
\text { comuns }\end{array}$ & $\begin{array}{l}\text { É realizada na } \\
\text { drogaria }\end{array}$ & $\begin{array}{l}\text { É realizada na } \\
\text { drogaria }\end{array}$ & $\begin{array}{l}\text { É realizada na } \\
\text { drogaria }\end{array}$ \\
\hline $\begin{array}{l}\text { Acondicionamento dos } \\
\text { resíduos comuns }\end{array}$ & $\begin{array}{l}\text { Em sacos plásticos } \\
\text { da cor preta }\end{array}$ & $\begin{array}{l}\text { Em sacos } \\
\text { plásticos da cor } \\
\text { preta }\end{array}$ & $\begin{array}{l}\text { Em sacos plásticos } \\
\text { da cor preta }\end{array}$ \\
\hline $\begin{array}{l}\text { Segregação dos resíduos } \\
\text { perfurocortantes }\end{array}$ & $\begin{array}{l}\text { São segregados na } \\
\text { drogaria }\end{array}$ & Não é realizada & Não é realizada \\
\hline $\begin{array}{l}\text { Acondicionamento dos } \\
\text { resíduos perfurocortantes }\end{array}$ & $\begin{array}{l}\text { Em recipientes } \\
\text { próprios para o } \\
\text { grupo E }\end{array}$ & Não é realizada & Não é realizada \\
\hline
\end{tabular}

Fonte: Pesquisa direta, 2018.

Como pode ser verificado no quadro acima, todas as empresas realizam os procedimentos conforme as etapas preconizadas pela legislação. A empresa 1 não realiza apenas a segregação e o acondicionamento de rejeitos radioativos. A empresa 2 também não realiza a segregação e o acondicionamento de rejeitos radioativos e relata não ter conhecimento sobre a segregação e o acondicionamento de resíduos biológicos e acondicionamento de resíduos químicos. Já a empresa 3 realiza apenas a segregação e o acondicionamento de resíduos comuns.

A segregação adequada exige a participação efetiva de toda a comunidade. Por esse motivo, todos os estabelecimentos devem conscientizar seus funcionários sobre a devida importância desse procedimento, mostrando os aspectos organizacionais como a subdivisão do estabelecimento de saúde, conforme os serviços especializados, classificação dos resíduos 
sólidos gerados e determinação de responsabilidades aos colaboradores (AMARANTE; RECH; SIEGLOCH, 2017).

A finalidade principal da segregação, não é a redução da quantidade de resíduos infectantes e sim de designar uma cultura organizacional de segurando e de não desperdício. Quando a segregação é realizada de forma correta, é possível melhorar a identificação da geração de resíduos e realizar uma decisão adequada para a redução e destinação (AMARANTE; RECH; SIEGLOCH, 2017).

Cussiol (2008) mostrou em seu estudo que a segregação dos resíduos de serviços de saúde é realizada apenas em $82 \%$ das empresas, em compensação $18 \%$ das empresas que não realizam essa etapa, acaba aumentando a quantidade, o volume e o risco ocasionado pelos mesmos. No atual estudo observou-se que o volume de resíduos contidos nas empresas estava de acordo com a capacidade de armazenamento, sendo um indicativo da eficiência do sistema de coleta.

Avaliou-se na presente pesquisa outras etapas do PGRSS, que está sendo relatada no quadro abaixo (quadro 2), separadas por empresas.

Quadro 2. Etapas do PGRSS realizadas nas empresas 1, 2 e 3.

\begin{tabular}{|l|l|}
\hline \multicolumn{2}{|c|}{ EMPRESA 1 } \\
\hline Identificação & Sim \\
\hline Transporte Interno & Carrinho com tampa \\
\hline Armazenamento & $\begin{array}{l}\text { Local exclusivo, separado dos produtos } \\
\text { farmacêuticos }\end{array}$ \\
\hline Tratamento Interno & Não \\
\hline Tratamento Externo & Sim \\
\hline Coleta Interna & Realizada por funcionário da empresa \\
\hline Coleta Externa & SERQUIP - Empresa terceirizada \\
\hline Transporte Externo & Veículo exclusivo \\
\hline Destinação Final & Aterro sanitário \\
\hline & \\
\hline Continua.. & \\
\hline
\end{tabular}




\begin{tabular}{|l|l|}
\hline \multicolumn{2}{|c|}{ EMPRESA 2 } \\
\hline Identificação & Sim \\
\hline Transporte Interno & Carrinho com tampa \\
\hline Armazenamento & DML \\
\hline Tratamento Interno & Não \\
\hline Tratamento Externo & Sim \\
\hline Coleta Interna & Realizada por funcionário da empresa \\
\hline Coleta Externa & SERQUIP - Empresa terceirizada \\
\hline Transporte Externo & Veículo exclusivo \\
\hline Destinação Final & Aterro sanitário \\
\hline \hline & EMPRESA 3 \\
\hline \hline Identificação & Sim \\
\hline Transporte Interno & Manual \\
\hline Armazenamento & DML \\
\hline Tratamento Interno & Não \\
\hline Tratamento Externo & Não tem conhecimento \\
\hline Coleta Interna & Realizada por funcionário da empresa \\
\hline Coleta Externa & Serviço de coleta pública \\
\hline Transporte Externo & Veículo de coleta de resíduo comum \\
\hline Destinação Final & Aterro sanitário \\
\hline
\end{tabular}

Fonte: Pesquisa direta, 2018.

Como pode ser observado, as três empresas seguem o PGRSS, realizando a identificação, o transporte, o armazenamento, o tratamento, a coleta e o transporte de forma correta, até que os resíduos cheguem na destinação final.

Nas três empresas analisadas, a coleta é realiza por colaboradores do local, assim como no estudo de Cussiol (2008). No entanto, esse estudo mostrou que os profissionais que atuam na coleta, não recebem o treinamento adequado para realizar tais procedimentos, gerando durante o manuseio dos resíduos um potencial risco de acidentes (CUSSIOL, 2008). 
Por esse motivo, o treinamento deve ser realizado pela empresa, apresentando informações básicas sobre a separação dos resíduos, as medidas de prevenção e higiene pessoal, além do treinamento de segregação, manuseio e acondicionamento dos resíduos. Dessa forma, torna-se notório a ausência de informações que são passadas das empresas para seus funcionários, podendo ocasionar problemas, principalmente para esses trabalhadores, pela falta de conhecimento das pessoas que desempenham funções relacionadas aos resíduos (MORESCHI, et al., 2014).

Em contraponto muitos professores, alunos e demais profissionais da área de saúde se preocupam e confirmam a necessidade de serem melhor informados e treinados em todas as informações sobre os resíduos. Nem sempre o manuseio é realizado de forma inadequada (SOUZA; ANDRADE, 2014). Dessa forma, o estudo avaliou o manejo interno dos resíduos produzidos em uma rede de drogarias no interior da Bahia de acordo com as normas brasileiras.

\section{Considerações Finais}

Os resultados obtidos apresentaram a situação das três empresas através da aplicação do questionário, e mostrou que cada estabelecimento tem um gerenciamento diferente, podendo ser ocasionado pela quantidade de resíduos gerados por cada empresa, através da sua composição, do acondicionamento correto, associado aos procedimentos de segregação e identificação que resguardem a sua contenção até o destino final, onde apresentaram resultados satisfatórios conforme preconizados pela legislação.

Tendo em vista atingir os principais objetivos das legislações vigentes, é de suma importância que seja definida estratégias adequadas para facilitar o manejo dos resíduos nos serviços de saúde, bem como a capacitação de todos os funcionários e profissionais envolvidos nesse processo, a fim de preservar o meio ambiente, prevenir, reduzir riscos ocupacionais e diminuir a quantidade de resíduos gerados.

Diante dessas perspectivas sobre o Gerenciamento resíduos dos serviços de saúde, não somente as empresas que foram avaliadas, mas a sociedade em geral, cabe a cada cidadão a 
tarefa de apontar as falhas, as atitudes corretas que devem ser observadas, no sentido de colaborar para que todos possam ter um ambiente saudável e uma melhor qualidade de vida.

\section{Referências}

ABNT, Associação Brasileira de Normas de Técnicas NBR 10.004: Resíduos sólidos Classificação. Rio de Janeiro. 2004, 77p.

ABNT, Associação Brasileira de Normas de Técnicas NBR 7.500: Símbolos de risco e manuseio para o transporte e armazenamento de resíduos sólidos, Identificação. Rio de Janeiro. $1887,47 \mathrm{p}$.

NBR 13853 - Coletores para resíduos de serviços de saúde perfurantes ou cortantes requisitos e métodos de ensaio, de maio de 1997.

BRASIL. Agência Nacional de Vigilância Sanitária. Resolução RDC $\mathbf{n}^{\mathbf{0}}$ 306, de 07 de dezembro de 2004. Dispõe sobre o Regulamento Técnico para o gerenciamento de resíduos de serviços de saúde. Publicada no Diário Oficial da União, Brasília (DF), 2004.

. Conselho Nacional do Meio Ambiente. Resolução no 358 de 29 de abril de 2005. Brasília, 2005. Dispõe sobre o Tratamento e a disposição final dos resíduos de serviços de saúde. Publicada no Diário Oficial da União, Brasília (DF), 2005.

Política Nacional de Resíduos Sólidos. (2010) Lei no 12.305, de 2 de agosto de 2010. Institui a Política Nacional de Resíduos Sólidos; altera a Lei $\mathrm{n}^{\circ}$ 9.605, de 12 de fevereiro de 1998; e dá outras providências. Publicada no Diário Oficial da União, Brasília (DF), 2010.

Ministério da Saúde. Manual de gerenciamento de resíduos de serviços de saúde Brasília: Ministério da Saúde, 2006.

ABRELPE. Associação Brasileira de Empresas de Limpeza Pública e Resíduos Especiais. Panorama dos resíduos sólidos Brasil. 2014.

AMARANTE, J. A. S.; RECH, T. D.; SIEGLOCH, A. E. Avaliação do gerenciamento dos resíduos de medicamentos e demais resíduos de serviços de saúde na Região Serrana de Santa Catarina. Revista Engenharia Sanitária Ambiental, v. 22, n. 2, p. 317-326, 2017.

ANVISA - Agência Nacional de Vigilância Sanitária. Resolução n ${ }^{a}$ 306, de 07 de dezembro de 2004. Dispõe sobre o Regulamento Técnico para o gerenciamento de resíduos de serviços de saúde. 2004. 
BUENO CS, WEBER D, OLIVEIRA KR. Farmácia caseira e descarte de medicamentos no bairro Luiz Fogliatto do município de Ijuí - RS. Rev Ciênc Farm Básica Apl. 2009;

CUSSIOL, N. A. de M. Manual de gerenciamento de resíduos de serviços de saúde. Belo Horizonte, 2008.

MAIA M, GIORDANO F. Estudo da situação atual de conscientização da população de santos a respeito do descarte de medicamentos. Rev. Ceciliana [homepage Internet] 2012.

MORESCHI, C.; REMPEL, C.; BACKES, D.S.; CARRENO, I.; SIQUEIRA, D.F.; MARINA, B. A importância dos resíduos de serviços de saúde para docentes, discentes e egressos da área da saúde. Revista Gaúcha de Enfermagem, v. 35, n.2, p. 20-26, 2014.

MORO, L, D.; PANDOLFO, A.; MARTINS, M. S.; GOMES1, A. P.; MACULAN, L. S. Abordagem do gerenciamento de resíduos de serviços de saúde em drogarias e farmácias de manipulação em cidade de pequeno porte da região sul do brasil. Holos Environment, v. 17, n. 1, p. 79-93, 2017..

SCHNEIDER, V. E. et al. Manual de Gerenciamento de Resíduos Sólidos de Serviços de Saúde. São Paulo: Balieiro, 2004, 173p.

SOUZA, C. L.; ANDRADE, C. S. Saúde, meio ambiente e território: uma discussão necessária na formação em saúde. Ciência e Saúde Coletiva, v. 19, n. 10, p. 4113-4122, 2014.

\section{Como citar este artigo (Formato ABNT):}

OLIVEIRA, Rafael Diego C. M.; COQUEIRO, Jaqueline F.R.; PORTELA, Fernanda S.; FERRAZ, Monique Alves. Avaliação do Plano de Gerenciamento de Resíduos dos Serviços de Saúde em uma rede de Drogarias de Vitória da Conquista-BA. Id on Line Rev.Mult. Psic., 2018, vol.12, n.40, p. 815-825. ISSN: 1981-1179.

Recebido: 23/01/2018

Aceito 24/05/2018 